\title{
An Automatic Tune-measurement system for the CELSIUS ring.
}

\author{
T. LOFNES \\ The Svedberg Laboratory, Uppsala, Sweden
}

\section{Abstract}

In CELSIUS [1] the tunes are up till now measured by FFTanalysing the response, at a beam position monitor, of a transverse kick of the beam. This FFT has to be performed over a wide frequency range and requires a high sampling frequency, and thus a large number of samples. By mixingdown the frequency range of interest, with a signal equal to the RF-frequency multiplied by the expected tune, and measure the deviation from the expected tune-value, the FFT can be made with a smaller number of samples and in the same frequency range independently of the RF-frequency. This way the FFT will be calculated faster by the computer, making it possible to measure the Q-values with a higher repetition rate. The set-up of this system and the results will be reported.

\section{INTRODUCTION}

In CELSIUS the Q-values are presently calculated in approx. 15 seconds. This is done with an FFT, in a digital oscilloscope, that is broadband $(0-50 \mathrm{MHz})$ and has a rather high frequency resolution $(2 \mathrm{kHz} /$ point) For this a sampling frequency of $100 \mathrm{MHz}$ is needed together with $50 \mathrm{k}$ of samples. To decrease the calculation time the number of samples has to be reduced. One way of doing this, without decreasing the frequency resolution, is to mix down the frequency range that contains the betatron sideband to a range close to $0 \mathrm{~Hz}$. This can be done (see Figure 1) by mixing the signal from the pick-up with a frequency equal to frev * qexp, where frev is the revolution frequency, $q$ is the fractional part of the tune $\mathrm{Q}$ and qexp is the expected value of $\mathrm{q}$, and measure the deviation of $\mathrm{q}$ from qexp.

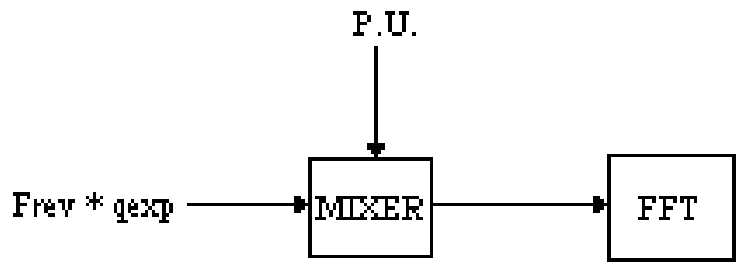

Figure 1

This mixed signal can be analysed by a FFT with a lower sampling frequency $(<1 \mathrm{MHz})$ and with less samples $(<1000)$. If $\mathrm{fd}$ is the frequency of this signal the Q-value will be: where $\mathrm{N}$ is the integer part of $\mathrm{Q}$.

With the same oscilloscope the FFT calculation time now will be less than 0.3 seconds.

\section{HARDWARE}

For each tune-measurement the beam is excited by a magnetic kicker. The resulting oscillation of the beam is measured by an electrostatic beam position monitor. The signal from this pick-up is amplified and low pass filtered and sent to an Image Rejection Mixer together with a signal of frequency $f$, $=$ frev $*$ qexp $-50 \mathrm{kHz}$, which is formed in a Direct Digital Syntesizer (see Figure 2). $50 \mathrm{kHZ}$ is subtracted in order to match the $0-100 \mathrm{kHz}$ range of the mixer.

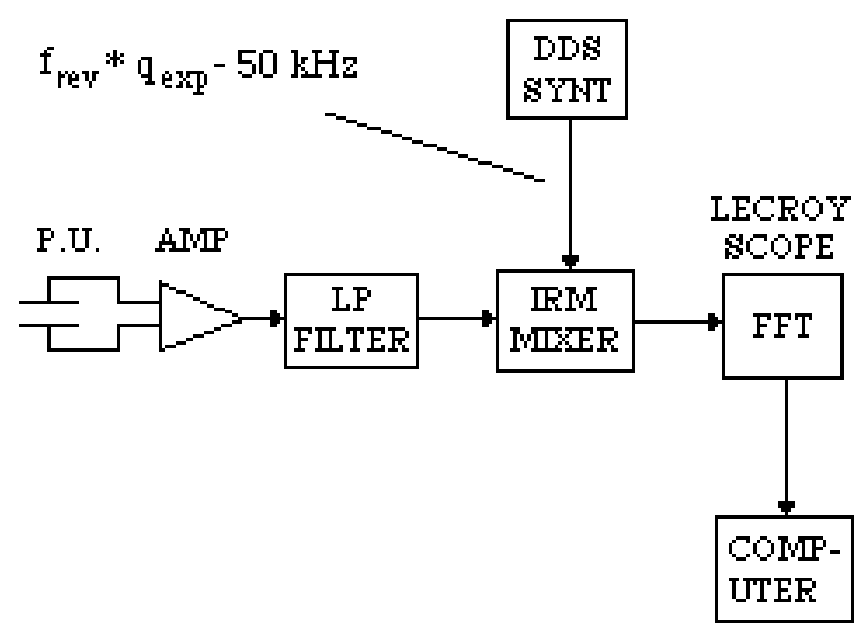

Figure 2. Hardware for the tune measurement system.

The sampling and the FFT calculation of the mixed signal is done by a LeCroy digital oscilloscope and the peak frequency in the FFT spectrum in each measurement is sent to the control computer for tune calculation and automatic correction.

$\mathrm{Q}=\mathrm{qexp}-\mathrm{fd} /$ frev $+\mathrm{N}$ 


\section{RESULTS}

Figure 3 and Figure 4 compares broadband FFT with FFT of the downmixed signal.

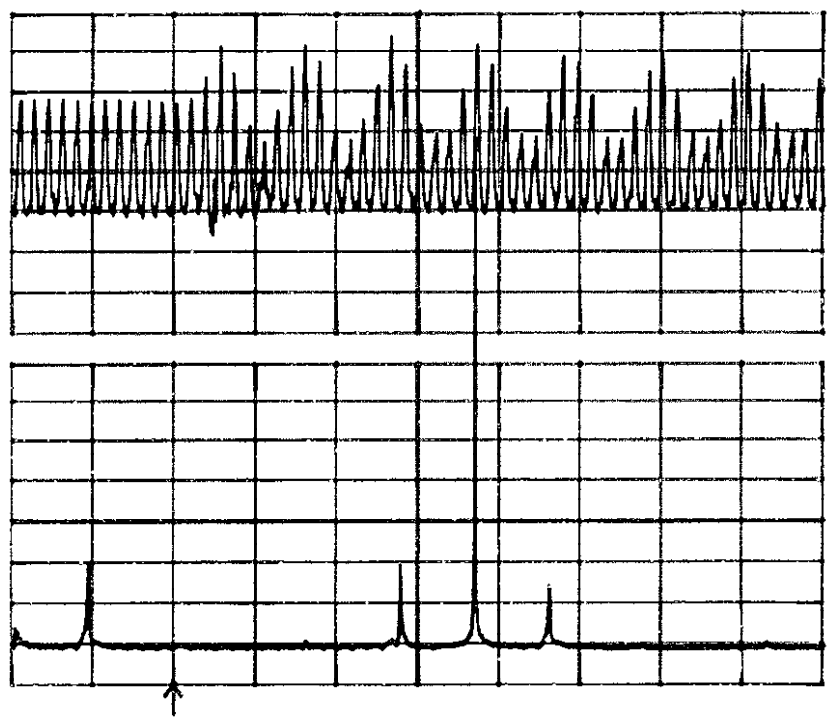

Figure 3. FFT of a broadband signal from the position monitor. Upper trace: pick-up signal, $10 \mu \mathrm{s} /$ div. Lower trace: FFT of upper trace (RF-frequency and 3 betatron sidebands are shown), $100 \mathrm{kHz} / \mathrm{div}$.

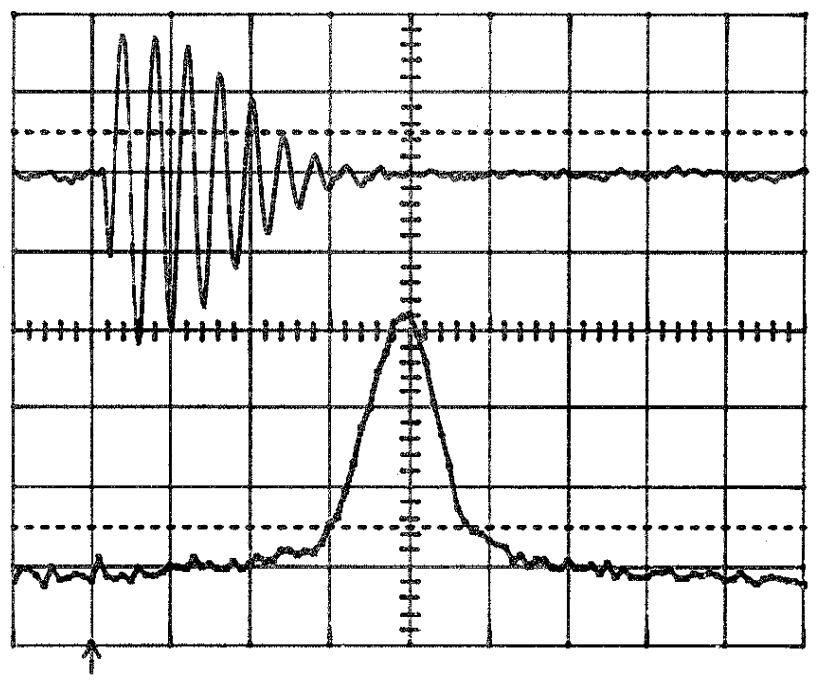

Figure 4. FFT of a downmixed signal. Upper trace: Downmixed signal, $50 \mu \mathrm{s} /$ div. Lower trace: FFT of upper trace, $10 \mathrm{kHz} / \mathrm{div}$.

The error in $\mathrm{q}$ is estimated to be +/- 0.001 in both measurements. With frequency interpolation the error will be even less [2]. The calculation time has been reduced from 15 seconds of the signal shown in Fig. 3 to less than 0.3 seconds of the signal shown in Fig. 4, and with faster processors the calculation time will be reduced to $3 \mathrm{~ms}$ [2].

\section{CONCLUSION}

With this method it is possible to measure the Q-values at least 2 times per second, which is fast enough in CELSIUS. With more computer power it is possible to do the measurements even faster.

\section{REFERENCES}

[1] T. Bergmark et al., Proc. 4th European Particle Accelerator Conference, London, England, 1994, 202

[2] J. Gonzalez, S. Johnston, and E. Schulte, "Fast Qmeasurement for the PS by FFT Analysis", Proc. 4th European Particle Accelerator Conference, London, 1994, 1734. 